To cite this article:

Kuppens, P. (in press). Improving theory, measurement, and reality to advance the future of emotion research. Cognition \& Emotion. 
RUNNING HEAD: Theory, measurement, and reality to advance the future of emotion research

Improving theory, measurement, and reality to advance the future of emotion research

Peter Kuppens

KU Leuven - University of Leuven, Belgium.

Total Word Count: 2535

Correspondence: $\quad$ Peter Kuppens

Faculty of Psychology and Educational Sciences

KU Leuven - University of Leuven

Tiensestraat, 102, box 3717, 3000 Leuven, Belgium

peter.kuppens@kuleuven.be

Acknowledgments: The writing of this manuscript was aided by funding from KU Leuven Research Council Grant GOA/15/003. 


\begin{abstract}
In this short contribution I reflect on three domains I think are important to advance emotion research. The first concerns theoretical progress, which I hope will occur in the form of theoretical unification that will allow for a consensual definition and understanding of its main object of study. The second concerns measurement where in parallel to technological advances to measure behavior and biology, particularly the measurement of experience deserves more attention. The third concerns reality, where I advocate the need to study real-world phenomena.
\end{abstract}

Keywords: emotion theory; measurement; ecological validity; daily life 


\section{Improving theory, measurement, and reality to advance the future of emotion research}

Leaving aside pre-experimental philosophers and thinkers, the scientific study of emotion is almost as old as the scientific study of psychology itself (Wundt, 1897/1998). Aside from illustrating the central place emotion takes for understanding the human mind and behavior, this relative long tradition of research on emotion may come with the expectation that surely this must be one of the domains that has made most progress and has generated a number of undisputed definitions, laws, and empirical phenomena or, in other words, certainties. And sure enough, emotion researchers do know a lot about emotions. They know and I hope agree that people's emotions are meant to guide them to adaptively cope with the world around them (Nesse \& Ellsworth, 2009), that emotions consequently arise as a result of the interaction (or more precisely perceived match or mismatch) between what happens in the environment and people's own (culture's) expectations, concerns, and values (Frijda, 2007); emotion researchers know that emotions involve responses in multiple systems, including physiology, behavior, cognition, and most dramatically conscious experience (Russell, 2003; Scherer, 2009), and that these responses in turn have important downstream consequences for how people perceive and interact with the world (Clore \& Huntsinger, 2007). These insights are not trivial, and are generative for the majority of contemporary research into emotion and affective phenomena.

Yet, at the same time, we are not there yet. Many issues about the nature, causes and consequences of emotions remain poorly understood. Below I discuss three domains in which advances would greatly help the progress of knowledge and insight in the study of emotion. These are situated at the level of theory, measurement, and relation to reality.

\section{Theory}

The domain of emotion research is (in)famously known for having as many definitions of emotions as there are emotion researchers (note by the way that the same can be said for many other fields, but still). To our personal dismay and public embarrassment, there is no widely accepted 
definition of the central object of our science (Scherer, 2005). I think that one of the crucial reasons for the lack of definition of emotion is that we lack a consensual theory of what emotions are and what causes emotions. A number of theoretical frameworks circulate in the field, the most important or widespread of which were featured in a recent issue of Emotion Review, namely appraisal theories of emotions (Moors, 2014), constructionist theories of emotions (Barrett, 2014; Mesquita \& Boiger, 2014), and evolutionary accounts of distinct emotions (Tracy, 2014). On the one hand, this could be viewed as affording emotion researchers the eclectic luxury of a number of frameworks, frames of thought, and approaches to emotion that could enrich our thinking and understanding. Yet, on the other hand it may also hold emotion researchers back in their science because it impedes defining our object of study, and allows for an eclectic reading of literature.

Arguably one of the most divisive issues is found in the debate on whether different emotion terms such anger fear, sadness, happiness can be considered to correspond to qualitatively different categories or mental processes, or whether these categories cannot be meaningfully distinguished from each other in any meaningful way (e.g., see Barrett, 2006 and Panksepp, 2007 as just one example of this debate). The tenacity of both parties and the longstanding nature of the debate could mean that the truth could lie somewhere in between. At least in my view there seems to be little convincing evidence that these different emotional labels rigidly co-occur with distinct physiological, neural, or behavioral signatures (Lindquist, Wager, Kober, Bliss-Moreau, \& Barrett, 2012; Siegel, et al., 2018) and that there is tremendous variability in these elements and their coherence across contexts and individuals. Yet, there also are convincing arguments to be made that there are qualitative differences between a number of motivational states in how we relate to the world that at least show some correlation with lay people's terms of these different emotional states (e.g., Frijda \& Parrott, 2011; Kreibig, 2010).

What I believe our science is in urgent need of, and my hope for the future, is the development of an overarching model that somehow is able to bring together the notion of a 
number of qualitatively different motivational states involved in emotional phenomena with the observations that there is great variability (across contexts, individuals, and cultures) in and between the eventual phenomenology of what we experience as emotional states, and that everyone can agree on and build on.

How could this be achieved? An effort from the entire field may be called for. One proposal could be to bring together the leading theorists in the field in a symposium that is aimed towards charting the agreements and disagreements between different positions, and agreeing on empirical efforts that would decide in areas of disagreement. Next a Manylabs project with researchers from around the world could repeat these experiments to identify universal patterns (within preestablished boundaries) and variability across emotion labels, individuals, cultures. The result could be a map of the emotion domain that charts areas of relative uniformity and areas of variability, which could form the basis for further theory development.

What could a resulting overarching theory look like? Likely, this could take the form of proposing that the emotional realm ranges from a limited number of motivational systems that have evolved to cope with specific environmental threats and opportunities (giving rise to basic fight/flight and approach behavioral tendencies) and that under certain conditions can be observed in relatively pure form but that however in many instances in normal daily life our emotional experiences are often constructed on top of those in the moment or even constructed de novo based on bodily sensations, situational information, memory, predictions of the world, and cultural values and meaning. Without an overarching model, we run the risk of getting stuck in almost ideological debates. With such a model, we can start testing it empirically.

\section{Measurement}

A necessary condition for good empirical science is good measurement. If emotion researchers are not able to adequately measure emotion, we will not be able to make much progress. While clearly major steps have been taken to advance in the measurement of emotion, still 
work is needed. I see two important areas that need particular attention. With the invention of increasingly sophisticated techniques to measure more "objective" signals of emotions, such as physiology, muscle movement, or neural activity, the risk arises that these techniques are considered as superior replacements of more old-school methods such as self-report for studying emotions ("can your finding not be backed up by using more objective measures of emotion!?"). Two considerations are of importance here. One, I think it has become abundantly clear that there is a relatively low correspondence across the board between the different modalities or components involved in emotion (Mauss \& Robinson, 2009; this links up with the abovementioned large variability within and between emotions). Thus, one modality cannot be considered to be a mere replacement or proxy of another modality, but rather reflects a different aspect or functionality of the emotion system.

Subjective experience of emotion and the language people use to describe and communicate it is not just one component of emotion that is interchangeable with another component, but rather represents the most defining element of emotion (see also, Lieberman, this issue). It therefore remains crucial to involve experience in the study of emotion and affective phenomena (where possible and meaningful).

This brings me to the second issue that is in need of attention. Emotion researchers need to work on better ways to measure emotional experience. The field has too long relied on the assumption that simple Likert-scale types of questions that show some internal consistency and meaningful correlations are sufficient. Yet, we know that such methods are susceptible to all kinds of bias and demand effects (due to poor or distorted memory and forecasting, social desirability, order and presentation effects, and so on). While millions are being spent on building better scanners and equipment to measure "objective" indicators of psychological phenomena, we could do with some more effort and similar devotion to improve the ways in which we measure (emotional) experience, without which we may not even speak of emotion. We need to refine self-report instruments such that biases known to affect such reporting are minimized (for a recent example, see for instance, 
Brown \& Maydeau-Olivares, 2013). Furthermore, we should keep looking for the exact conditions and circumstances under which other channels (behavior, peripheral, physiology, neural processes) may provide a more or less direct read-out of experience, even though this seems more challenging than perhaps initially hoped.

\section{Reality}

Theories and measurement are indispensable but in the end they need to be able to say something about what people do, say, feel, need, want, love, and hate in real life. The importance of experimental research is undeniable in the field of psychology and it is impossible to underestimate its value for the advancement of our science. Yet, at the same time, we also need to, or perhaps even have a moral obligation to at some point verify and confirm that the behavior we study on a keyboard, in a lab, and inside a scanner is in fact relevant to what takes place in people's everyday lives and relates to real-world phenomena, or can contribute to solving real world problems or creating novel opportunities. This pursuit can be (and is) achieved in many different degrees and forms and at many different levels. We can make sure we study stimuli and outcomes that take a real place in people's lives (e.g., Coo, Milgrom, Kuppens, \& Trinder's 2015 study studies emotions and appraisals in the context of childbirth). We can include in our research the observation of real-life behavior, choices, likes and dislikes (e.g., Pe, Gotlib, Van den Noortgate, \& Kuppens' 2016 study social rejection in the context of a real-life speed dating context). We can track people's anticipation, responses, and regulation towards real events that take place in their lives (e.g., Kalokerinos, Erbas, Ceulemans, \& Kuppens' 2018 study examines students' anticipations, responses, and regulation in relation to receiving their exam grades in $1^{\text {st }}$ year of higher education). While for the experimentallyinclined researcher, such efforts may imply coming to terms with letting go some of the rigorous control of lab experiments, I would hope it may come with the satisfaction of gaining real-world relevance.

\section{Concluding Remarks}


In many ways, I believe emotion research is way ahead of many of its peers. Its focus on one common (albeit hard to define) psychological phenomenon, be it as object in itself or cause or consequence of something else, sets it apart from many of the other traditional subfields that often focus on a very broad class of determinants of behavior (social psychology, personality psychology, developmental psychology, biological psychology, etc.). As a result, emotion research is not stuck in one specific paradigm, frame of thinking, or method. Rather, itis able to presents itself as a very diverse and versatile scientific area that takes advantage of diverse approaches and methods. The focus on one topic gives emotion research unity and at the same time affords it diversity. Theoretical unification, improvement of measurement, and real-world relevance will allow it to arrive at a consensual definition of its object, initiate concerted efforts to pinpoint its universal elements and determinants of its variation across context and individuals, and consequently formulate informed advice on how to change emotions to improve people's lives. In short, it would help it to become the scientific discipline it deserves to be. 


\section{References}

Barrett, L. F. (2006). Are emotions natural kinds?. Perspectives on Psychological Science, 1, 28-58.

Barrett, L. F. (2014). The conceptual act theory: A précis. Emotion Review, 6, 292-297.

Brown, A., \& Maydeu-Olivares, A. (2013). How IRT can solve problems of ipsative data in forcedchoice questionnaires. Psychological Methods, 18, 36.

Clore, G. L., \& Huntsinger, J. R. (2007). How emotions inform judgment and regulate thought. Trends in Cognitive Sciences, 11, 393-399.

Coo, S., Milgrom, J., Kuppens, P., \& Trinder, J. (2015). Perinatal distress: An appraisal perspective. Journal of Reproductive and Infant Psychology, 33, 190-204.

Frijda, N. H. (2007). The laws of emotion. Mahwah, NJ: Erlbaum.

Frijda, N. H., \& Parrott, W. G. (2011). Basic emotions or ur-emotions?. Emotion Review, 3, 406-415.

Kalokerinos, E. K., Erbas, Y., Ceulemans, E., \& Kuppens, P. (2018). Emotion differentiation and emotion regulation in daily life. Manuscript submitted for publication

Kreibig, S. D. (2010). Autonomic nervous system activity in emotion: A review. Biological Psychology, 84, 394-421.

Lindquist, K. A., Wager, T. D., Kober, H., Bliss-Moreau, E., \& Barrett, L. F. (2012). The brain basis of emotion: a meta-analytic review. Behavioral and Brain Sciences, 35, 121-143.

Mauss, I. B., \& Robinson, M. D. (2009). Measures of emotion: A review. Cognition and Emotion, 23, 209-237.

Mesquita, B., \& Boiger, M. (2014). Emotions in context: A sociodynamic model of emotions. Emotion Review, 6, 298-302.

Moors, A. (2014). Flavors of appraisal theories of emotion. Emotion Review, 6, 303-307.

Nesse, R. M., \& Ellsworth, P. C. (2009). Evolution, emotions, and emotional disorders. American Psychologist, 64, 129-139.

Panksepp, J. (2007). Neurologizing the psychology of affects: How appraisal-based constructivism and basic emotion theory can coexist. Perspectives on Psychological Science, 2, 281-296. 
Pe, M. L., Gotlib, I. H., Van den Noortgate, W., \& Kuppens, P. (2016). Revisiting depression contagion as a mediator of the relation between depression and rejection: A speed-dating study. Clinical Psychological Science, 4, 675-682.

Russell, J. (2003). Core affect and the psychological construction of emotion. Psychological Review, $110,145-172$.

Scherer, K. R. (2005). What are emotions? And how can they be measured?. Social Science Information, 44, 695-729.

Scherer, Klaus R.(2009). The dynamic architecture of emotion: Evidence for the component process model. Cognition \& Emotion, 23, 1307-1351.

Siegel, E. H., Sands, M. K., Van den Noortgate, W., Condon, P., Chang, Y., Dy, J., ... \& Barrett, L. F. (2018). Emotion fingerprints or emotion populations? A meta-analytic investigation of autonomic features of emotion categories. Psychological Bulletin, 144, 343.

Tracy, J. L. (2014). An evolutionary approach to understand distinct emotions. Emotion Review, 6, 308-312.

Wundt,W. M. (1998). Outlines of psychology (C. H. Judd, trans.). Bristol, UK: Thoemmes Press. (Original work published 1897) 